\title{
Higgs mass and gravity waves in standard model false vacuum inflation
}

\author{
Alessio Notari ${ }^{*}$ \\ Dipartimento di Fisica, Università di Ferrara and INFN Sez. di Ferrara, Via Saragat 1, I-44100 Ferrara, Italy \\ and Departament de Física Fondamental i Institut de Ciències del Cosmos, Universitat de Barcelona, \\ Martí i Franquès 1, 08028 Barcelona, Spain
}

(Received 2 September 2014; published 23 March 2015)

\begin{abstract}
In previous publications we have proposed that inflation can be realized in a second minimum of the standard model Higgs potential at energy scales of about $10^{16} \mathrm{GeV}$, if the minimum is not too deep and if a mechanism which allows a transition to the radiation dominated era can be found. This is provided, e.g., by scalar-tensor gravity models or hybrid models. Using such ideas we had predicted the Higgs boson mass to be of about $126 \pm 3 \mathrm{GeV}$, which has been confirmed by the LHC, and that a possibly measurable amount of gravity waves should be produced. Using more refined recent theoretical calculations of the renormalization group equations we show that such scenario has the right scale of inflation only for small Higgs mass, lower than about $124 \mathrm{GeV}$, otherwise gravity waves are overproduced. The precise value is subject to some theoretical error and to experimental errors on the determination of the strong coupling constant. Finally we show that introducing a moderately large nonminimal coupling for the Higgs field the bound can shift to larger values and be reconciled with the LHC measurements of the Higgs mass.
\end{abstract}

DOI: 10.1103/PhysRevD.91.063527

PACS numbers: 98.80.Cq, 04.30.-w, 14.80.Bn

In [1-3] we have proposed that inflation in the early Universe can be realized for a narrow band of values of the top quark and Higgs boson masses, for which the standard model (SM) Higgs potential develops a second local minimum [4-8] at energy scales of about $10^{16} \mathrm{GeV}$. Such a scenario is viable if a successful transition to a radiation-dominated era can be obtained, which we have shown to be possible in Ref. [1] using an explicit model in the framework of a scalar-tensor theory of gravity developed in $[9,10]$ and in [3] using a hybrid inflationary model with an additional weakly coupled scalar, in standard gravity. Such a generic scenario could be realized only for a Higgs mass $m_{H}$ in the range $m_{H}=$ $(126.0 \pm 3.5) \mathrm{GeV}$, the error being mainly due to the theoretical uncertainty of the 2-loops renormalization group equations (RGE) used in that calculation. Such a prediction for the Higgs mass range has turned out to be surprisingly compatible with the measurement of a Higgs mass of $125.9 \pm 0.4 \mathrm{GeV}$ by the LHC [11,12].

As it is well known, inflation can generate quantum mechanically tensor (gravity wave) modes, usually parametrized through $r$, the ratio of tensor-to-scalar perturbation spectra at large scales. In $[1,2]$ we had predicted a relatively large amplitude for $r$ in our scenario, which is set by the overall height of the second minimum, which can be computed up to the precision of the RGE calculations and of the experimental input for the standard model parameters: $m_{H}$, the top mass $m_{t}$, and the strong coupling constant $\alpha_{s}$. However the situation has recently changed, since more refined theoretical calculations of the standard

notari@fe.infn.it model potential have been performed, therefore making more precise the correspondence between the input parameters and the scale of the second minimum. Recently the tensor modes had been claimed to be measured by the BICEP2 collaboration [13] with a large amplitude, which corresponds to a scale of about $2 \times 10^{16} \mathrm{GeV}$, but a more recent analysis [14] has shown that such a claim is invalid and only an upper bound at that scale can be set.

In this paper we update our results, using the results of [6,7] for the Higgs effective potential, and show that if the theoretical errors are under control the scale of inflation is generically larger than $2 \times 10^{16} \mathrm{GeV}$ and can be reconciled with experiments only if $m_{H} \lesssim 124 \mathrm{GeV}$ (plus a theoretical error, at present estimated to be of order $0.3 \mathrm{GeV}$ ). Similar conclusions have been recently found by [15] in the case of inflation from the Higgs false vacuum with an additional scalar weakly coupled to the Higgs, in standard gravity. Of course such conclusion can be changed if extra ingredients are added to the standard model. We show the results that can be obtained by introducing a nonminimal coupling of the Higgs to gravity. Of course less minimal modifications, such as a direct coupling of the Higgs to an extra scalar, can also easily shift the scale of inflation. Finally, provided the scale of inflation due to the Higgs potential is low enough, we also reanalyze the specific model based on scalar tensor theories [1], which realizes the exit to a radiation dominated era, showing that it could reproduce observations on $r$ and on the scalar spectral index $n_{s}$.

As in [1-3] we consider the potential for the Higgs field $\chi$ in the SM of particle physics. For very large values of the Higgs field such potential can be written as

$$
V(\chi) \simeq \lambda_{\mathrm{eff}}(\chi) / 4 \chi^{4}
$$


where $\lambda_{\text {eff }}$ is some effective quartic coupling [6] which is very close, but not identical, to the quartic coupling $\lambda$. Due to the running of couplings $\lambda_{\text {eff }}$ can become very small at high energies, while keeping always positive values (stability regime). In such conditions the potential can have an additional minimum at very high field values. While this is at present disfavored by the current calculations and measurements in the SM it still not ruled out. In particular this can still happen if the top quark mass has a low value compared to the present best fit $[16,17]$.

If the Higgs field starts in the false minimum at $\chi_{0}$ and dominates the energy density of the Universe, the Friedmann equation leads to a stage of inflationary expansion

$$
H^{2} \simeq \frac{V\left(\chi_{0}\right)}{3 M^{2}} \equiv H_{I}^{2}, \quad a(t) \propto e^{H_{I} t}
$$

where $a(t)$ is the scale factor, $H \equiv \dot{a} / a$ is the Hubble rate and $M$ is the reduced Planck mass $\left(M \equiv 2.435 \times 10^{18} \mathrm{GeV}\right)$.

A nontrivial model-dependent ingredient is a mechanism to achieve a graceful exit from inflation, that is a transition to a radiation-dominated era, in a nearly flat Universe at a sufficiently high-temperature. In [1] we have proposed that the Higgs field can tunnel to the other side of the potential barrier by nucleating bubbles [18] that can successfully collide and percolate, in the presence of a scalar tensor theory of gravity. Alternatively in [3] we had proposed that a smooth transition may happen also in standard gravity, in the presence of an extra scalar field very weakly coupled to the Higgs field. The main point of both mechanism is that they do not affect appreciably the SM runnings of couplings, since in the first model there is only a gravitational coupling to the standard model, and in the second case the dimensionless coupling constant is extremely small, of order $10^{-11}$. For this reason precise connections with low energy parameters still hold even in the presence of such new fields. Of course it is possible that such a new scalar can also interact with the Higgs field with a large direct coupling and in this case this could also affect the RGE equations and we briefly discuss later such possibilities.

Subsequently the Higgs field could roll down the potential, reheat the Universe, and finally relax in the present true vacuum with $v=246 \mathrm{GeV}$. In the scalartensor theory of gravity model an extra scalar field $\phi$ is introduced, the Brans-Dicke scalar or dilaton, which has an interaction term of the form $f(\phi) R$, where $R$ is the Ricci scalar and $f(\phi)>0$ thus sets the value of the Planck mass. The presence of such field makes the Planck mass timedependent, and therefore also $H$ during Inflation: this slows down the expansion sufficiently enough if $f(\phi)$ grows faster than $\phi^{2}$ for large $\phi$ values, since a stage of quasiexponential expansion is followed by a stage of power-law (even decelerated) expansion $[9,10]$. During the exponential phase the quantum fluctuations in $\phi$ lead to a nearly scale-invariant spectrum of perturbations. During the subsequent decelerated phase, $H$ decreases rapidly, and therefore the expansion is sufficiently slowed down, so that many bubbles can be nucleated in a Hubble patch and subsequently collide and reheat the Universe, leading to a spatially flat radiation dominated Universe.

As discussed in [1], after tunneling we require the field $\phi$ to relax to zero if a suitable potential $U(\phi)$ is present, which allows us to identify the present reduced Planck mass with the quantity $M$ and, at the same time, to satisfy constraints from fifth-force experiments and time-dependence of the Newton constant $G_{N}=1 /\left(8 \pi M^{2}\right)$ [19]. We will discuss this issue further in the present paper.

An alternative to scalar-tensor theories is given by models [3] with a direct tiny coupling of the Higgs field to an additional scalar field, which induces a timedependence directly into $\Gamma$ by flattening the barrier in the potential.

It is crucial to note that a graceful exit can be realized in the above models only if there is a very shallow false minimum, otherwise the tunneling rate would be negligibly small in the scalar-tensor model, since the probability is exponentially sensitive to the barrier [18]. Such shallowness is required also in the hybrid model since a change in the barrier by a large amount would introduce a departure from an almost flat scalar spectral index. So, the shape of the potential is very close to the case in which there is just an inflection point and thus we have a generic prediction for the scale of inflation and for $r$, while the specific model only affects the prediction for the spectral index of cosmological density perturbations $n_{S}$.

Using the recent state-of-the-art theoretical calculations given in [6,7], we show the specific values of the top and Higgs masses which allow for the presence of a false minimum. Such calculations are now so accurate that also the precise value of $\alpha_{3}\left(m_{Z}\right)$ inside the present allowed experimental range $\alpha_{3}=0.1184 \pm 0.007$ [16] is a relevant parameter. As an example, in Fig. 1 we also show the Higgs potential for very specific values of $m_{t}$ and $m_{H}$, showing agreement with Fig. 7 of [6]. The extremely precise values shown in the caption are not to be taken sharply, because of a theoretical uncertainty, estimated to be of about $0.3 \mathrm{GeV}$ on $m_{H}$ and about $0.15 \mathrm{GeV}$ on $m_{t}$.

Increasing (decreasing) $m_{t}$, one has also to increase (decrease) $m_{H}$ in order to develop the shallow false minimum; accordingly, the value of both $V\left(\chi_{0}\right)$ and $\chi_{0}$ increase (decrease). The solid blue lines in Fig. 2 show the points in the $m_{t}-m_{H}$ plane where the shallow SM false minima exists, for different values of $\alpha_{3}\left(m_{Z}\right)$ in the 3-sigma range. The red dashed lines show the regions in $\left(m_{H}, m_{t}\right)$ which corresponds to a value of $r$ compatible with observations, directly given by the height of the potential via the equation $r=\frac{V\left(\chi_{0}\right)}{\left(2.24 \times 10^{16} \mathrm{GeV}\right)^{4}} 0.2$ and it turns out that these lines point to a too small value of $m_{H}$ compared to the present experimental values. Of course this is a valid 
HIGGS MASS AND GRAVITY WAVES IN STANDARD ...

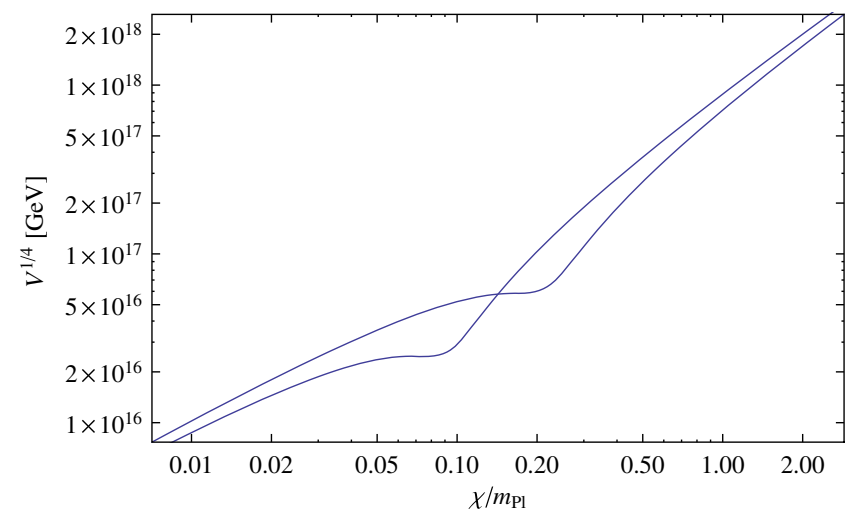

FIG. 1 (color online). Higgs potential as a function of the Higgs field $\chi$ in units of $m_{P l}=1.22 \times 10^{19} \mathrm{GeV}$. We fixed $\alpha_{3}\left(m_{Z}\right)=0.1184$. The upper curve correspond to $m_{H}=125$, $m_{t}=171.0305$, which shows good agreement with the results of [6], fig. 7. The lower curve corresponds to $m_{H}=123.2$, $m_{t}=170.1228$, and a value of $r=0.28$. As mentioned in the text and discussed in [1], in order to have a sizable tunneling probability through the left side, the barrier must be very low, almost as in an inflection point. Note that the value of the potential at the inflection point correctly reproduces Fig. 7 of [6]. We disagree with the recent update on the scenario of the present paper given in [20] immediately after the BICEP2 claims. The reader can check that Fig. 2 of such update does not reproduce Fig. 7 of [6], leading to a conclusion which underestimates $r$ by more than 2 of magnitudes.

conclusion under the assumption that the theoretical errors on $m_{H}$ are now estimated to be of about $0.3 \mathrm{GeV}$ by [6,7]. This scenario could still be compatible with data only if the theoretical errors in the RGE and the matching conditions were larger, say of about $1 \mathrm{GeV}$. $^{1}$

Discarding such possibility we show that this could be cured by introducing a nonminimal coupling $\xi \chi^{2} R$ also for the Higgs field, similarly to what was proposed by $[23,24]$, albeit in a different class of models. This transforms the potential in the slow roll phase in the Einstein frame in the following form (see [9] and citations therein):

$$
V(\chi)=\frac{\lambda_{\mathrm{eff}} / 4 \chi^{4}}{\left(1+\xi\left(\chi^{2} / M^{2}\right)\right)^{2}}
$$

which suppresses the potential at large field values and we show in Fig. 3 that a large value of $\xi$ allows for the potential to fit $\mathrm{CMB}$ observations, also for larger $m_{H}$, compatible

\footnotetext{
${ }^{1}$ Note also that such a conclusion is in agreement with [21], which also concludes that the height of the potential is too high with the present values of $m_{H}$ and $m_{t}$. For instance Fig. 4 of such paper shows that only very low $m_{H}$ or very high $\alpha_{s}$ (more than 3 sigma from the present measured values) would lead to acceptable values of $r$. Note also that [21] performs the analysis of the number of efolds, $n_{s}$ and $r$, only for the hybrid type of model introduced in [3], while in the present paper we focus on the scalar-tensor type of model introduced in [1].
}

PHYSICAL REVIEW D 91, 063527 (2015)

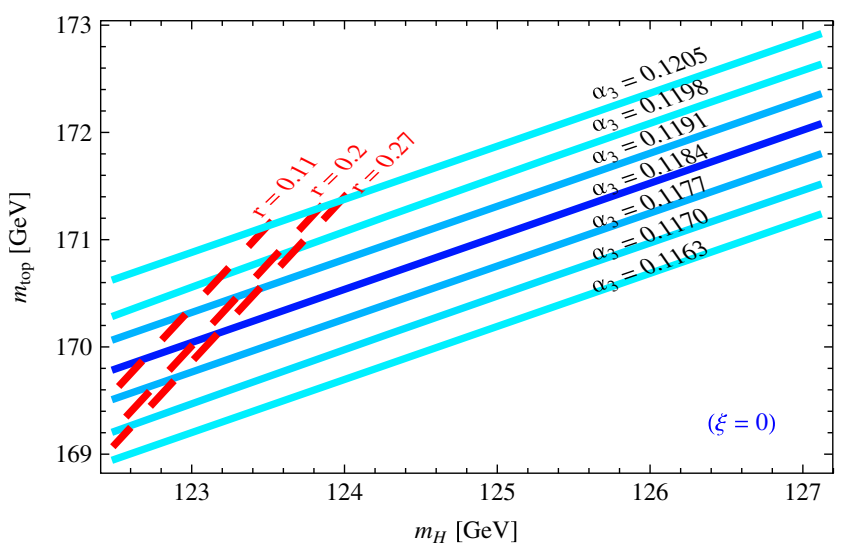

FIG. 2 (color online). The solid lines indicate the $m_{t}-m_{H}$ values compatible with a shallow Higgs false minimum, taking a $3 \sigma$ variation of $\alpha_{s}\left(m_{Z}\right)$ around its central value of 0.1184 . Here the Higgs is assumed to be minimally coupled $(\xi=0)$. There is an uncertainty of about $0.15 \mathrm{GeV}$ in $m_{t}$ and a horizontal one of $0.3 \mathrm{GeV}$ in $m_{H}$ due to the present $[6,7]$ theoretical uncertainties in the RGE. The red dashed lines represent the values of the tensorto-scalar ratio associated to the energy scale of the minimum, where we displayed the $r=0.2_{0.09}^{+0.07}$ values which correspond to the early claims by BICEP2 [13]. However since such a claim has been shown to be wrong by [14] and only an upper bound $r<0.12(95 \%$ CL) can be set. The present experimental values for the parameters are $m_{H}=(125.7 \pm 0.4) \mathrm{GeV}$, $m_{t}=(173.21 \pm 0.51 \pm 0.71) \mathrm{GeV}, \alpha_{3}=0.1185(6)$ [22].

with the LHC measurements. However let us comment that this is based only on a tree level analysis and when having a value of the extra coupling constant $\xi$ larger than $\mathcal{O}(1)$ we have to be careful about the regime of validity of the theory, similarly to the models proposed in [25], due to the fact that a new cutoff scale appears, $M_{P l} / \xi$. If such scale is higher than the energy scale of inflation, given by $V^{1 / 4}$ this should make the model consistent. Such a situation is not easy to achieve, but it is better fulfilled if $m_{H}-m_{t}$ are small and $\alpha_{3}$ is large, which allows $\xi$ to have a lower value. The discussion about consistency of this kind of models after quantum corrections includes the fact that the cutoff scale is field-dependent and it has been discussed by several papers (see, e.g., [26-29]).

Finally we also show the results for the $n_{s}-r$ plot for the scalar tensor model proposed in [1] in Fig. 4. Such a model is based on the introduction of an additional nonminimally coupled field $\phi$ with the following action:

$-S=\int d^{4} x \sqrt{-g}\left[\mathcal{L}_{\mathrm{SM}}+\frac{\left(\partial_{\mu} \phi \partial^{\mu} \phi\right)}{2}-\frac{M^{2}}{2} f(\phi) R-U(\phi)\right]$

where $\mathcal{L}_{\mathrm{SM}}$ is the standard model Lagrangian and the potential $U$ is not specified and assumed to be relevant only after inflation to stabilize the field. The coupling function is given by: 

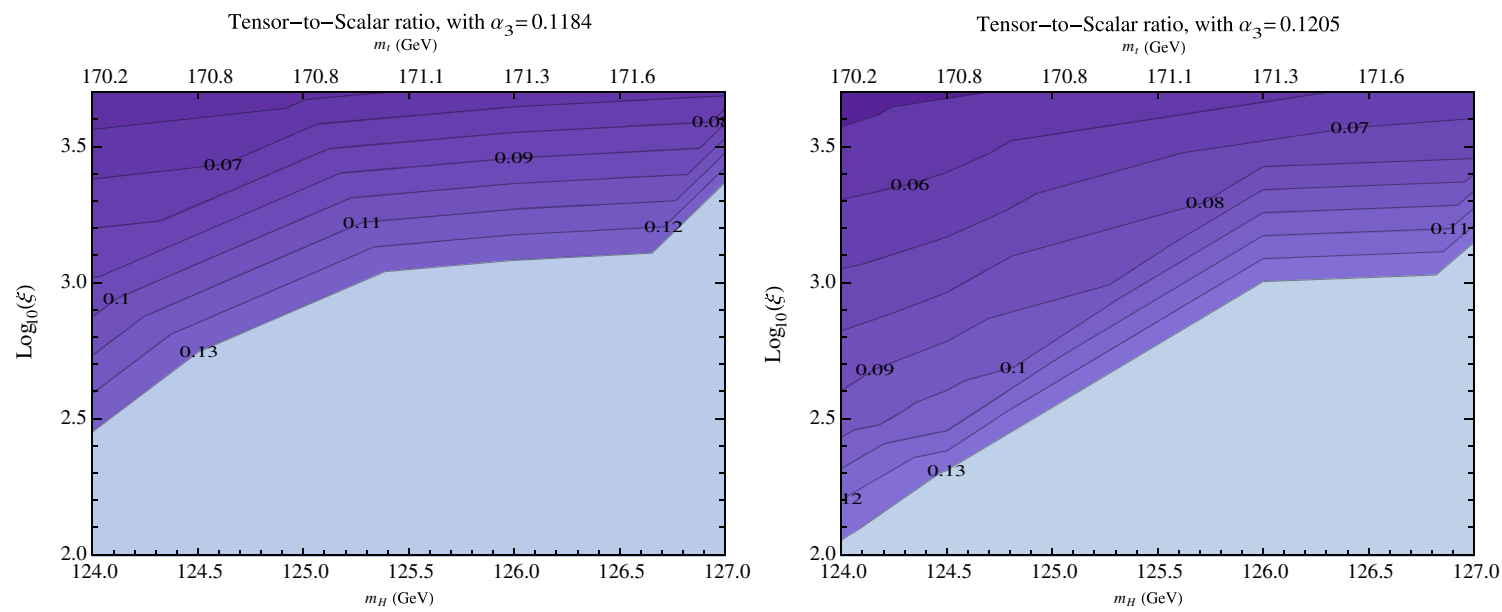

FIG. 3 (color online). The contours indicate different values of the tensor-to-scalar ratio $r$ associated to the energy scale of the minimum when introducing a the nonminimal coupling $\xi$; we show it as a function of the Higgs and top mass for the central value of $\alpha_{3}$ (left plot) and for a larger value ( $3 \sigma$ deviation, right plot). There is a (vertical) uncertainty of about $0.15 \mathrm{GeV}$ in $m_{t}$ and a (horizontal) one of $0.3 \mathrm{GeV}$ in $m_{H}$ due to the present [6,7] theoretical uncertainties in the RGE.

$$
f(\phi) \simeq 1+\beta\left(\frac{\phi}{M}\right)^{2}+\sum_{n \geq 4} \gamma_{n}\left(\frac{\phi}{M}\right)^{n}
$$

with the requirement that $f(\phi)>\phi^{2}$, which is guaranteed for instance if the couplings $\gamma_{n}$ are positive. Under these conditions $H(t)$ starts almost constant and after a sufficient umber of efolds starts decreasing as a power law, allowing for a tunneling transition when $H^{4}$ becomes equal to the tunneling rate per unit volume $\Gamma$. As a simple example we will study the case in which $\gamma_{4}>0$ and all other couplings are vanishing. If the Higgs fields is at the minimum and it is not evolving during inflation the only time dependent quantity is $\phi$ and so we can go the Einstein frame with a metric defined by $\bar{g}_{\mu \nu} \equiv f(\phi) g_{\mu \nu}$ and study the evolution of a canonically normalized field $\Phi$ defined through $d \Phi=d \phi \sqrt{K(\phi)}$, where

$$
K(\phi) \equiv \frac{2 f(\phi)+3 M^{2} f^{\prime 2}(\phi)}{2 f^{2}(\phi)} .
$$

In this frame the action becomes:

$$
S_{E}=\frac{1}{2} \int d^{4} x \sqrt{-\bar{g}}\left[M^{2} \bar{R}-(\bar{\partial} \Phi)^{2}-2 \overline{\mathcal{L}}_{\mathrm{SM}}\right]
$$

where the bar represents quantities in the Einstein frame. The Higgs potential contained in $\overline{\mathcal{L}}_{\mathrm{SM}}$ is now $V(\chi) / f(\Phi)^{2}$, so that the potential energy at the false Higgs minimum $\chi_{0}$ gives rise to a potential term for $\Phi$

$$
S_{E}^{\chi_{0}}=\int d^{4} x \sqrt{-\bar{g}} \bar{V}, \quad \bar{V} \equiv \frac{V\left(\chi_{0}\right)}{f(\Phi)^{2}} .
$$

This acts as a hill-top potential for the $\Phi$ field and we assume that $\Phi$ rolls down the potential from small to high values. Given the potential we define as usual the slow-roll parameters

$$
\epsilon(N)=\frac{1}{2}\left|\frac{1}{\bar{V}} \frac{d \bar{V}}{d(\Phi / M)}\right|^{2} \quad \eta(N)=\frac{1}{\bar{V}} \frac{d^{2} \bar{V}}{d(\Phi / M)^{2}}
$$

We solve numerically the Klein-Gordon equation for $\Phi$ under such a potential assuming that it starts as close to zero as possible [i.e., with an initial value $\Phi_{I}$ given by the quantum fluctuations $\left.\Phi_{I}=\bar{H} /(2 \pi)\right]$ together with the Friedmann equation, which gives us a numerical solution for $\Phi(N)$, where $\mathrm{N}$ is the number of efolds at some value of the scale factor $\bar{a}$, defined as $N \equiv \ln \left(\bar{a}_{F} / \bar{a}\right)$, where $\bar{a}_{F}$ is the scale factor at the end of the inflationary stage, defined by

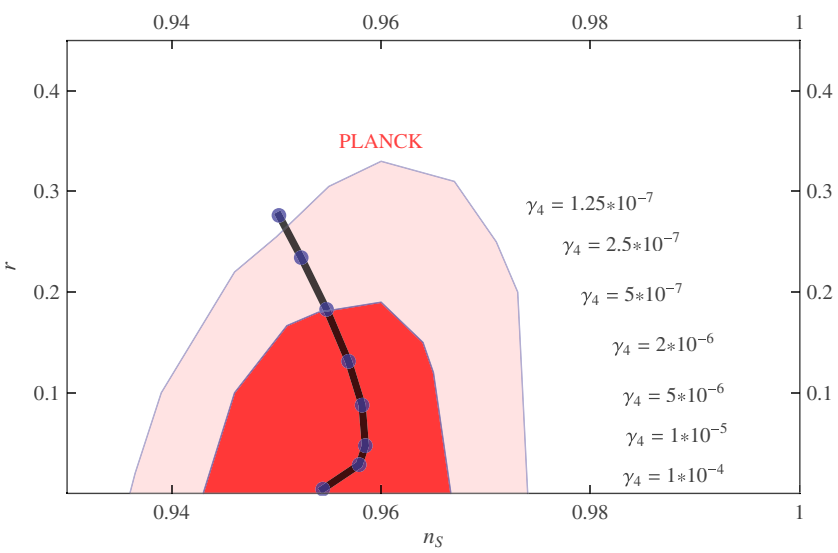

FIG. 4 (color online). Spectral index $n_{S}$ and tensor-to-scalar ratio $r$ for the scalar-tensor model of [1]. Here the number of efolds is assumed to be $\bar{N}=60$. Only one nonvanishing parameter $\gamma_{4}$ was assumed to be present in Eq. (4) and the various points along the black lines correspond to different values of $\gamma_{4}$. 

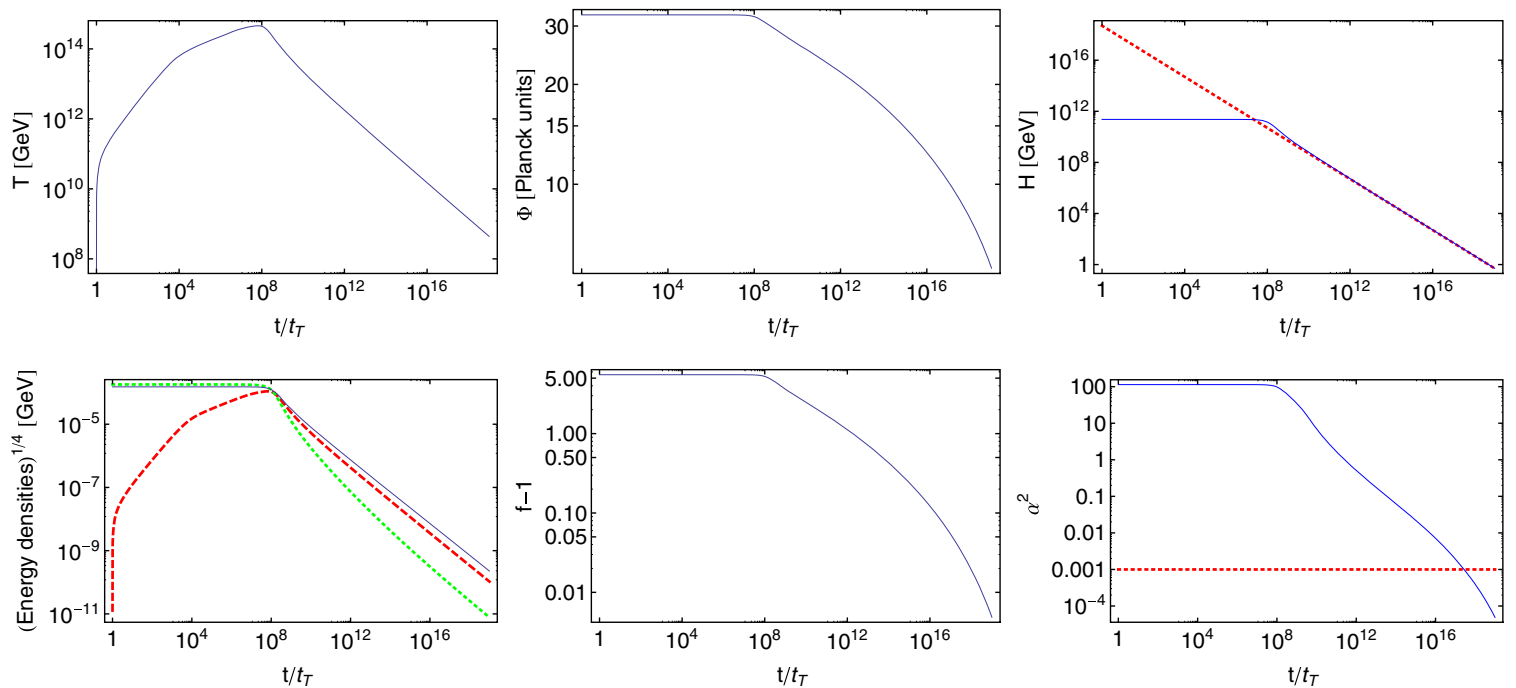

FIG. 5 (color online). Evolution after tunneling for a specific example assuming that the Higgs field starts at rest at a value $\chi_{T}=0.6 \chi_{0}$ and with a decay rate $\Gamma_{\chi}=10^{-2} \chi_{T}$. We have also chosen $\gamma_{4}=5 \times 10^{-6}$, and the field $\Phi$ is taken to start at rest at a value $\Phi / M=32$ : this would correspond to a decay happening when the Hubble rate $H_{T}$ is equal to the tunneling rate $\Gamma$, with the condition that $\Gamma$ is smaller than the inflationary Hubble rate $H_{I}$ by a factor $\left(\Gamma / H_{I}\right)^{4}<10^{-7}$ which ensures that no large bubbles are produced during inflation and subsequently imprinted in the CMB [10]. We used here a potential $U(\Phi)=\mu^{4}\left(e^{2 \Phi}-1\right)$ with $\mu=10^{8} \mathrm{GeV}$. The upper left panel shows the evolution of the temperature produced by the decay of the Higgs, the upper middle panel shows the evolution of the field $\Phi$, and the upper right panel shows the Hubble rate (solid line) compared to the Hubble rate during radiation domination $H=1 /(2 t)$ (red, dashed). The bottom left plot shows the relative energy densities in the potential $V(\chi) / f(\Phi)^{2}$ (green, dotted), in the potential $U(\Phi)$ (blue solid) and in radiation $\rho_{R}$ (red dashed). The bottom middle plot shows the evolution of $f-1$, which is proportional to the difference between the effective Planck mass and $M$. Finally the bottom right panel shows the quantity $\alpha^{2}$ of Eq. (A2) (solid blue), compared to the bound coming from fifth force experiments of about $10^{-3}$ (red, dashed).

either $\epsilon$ or $\eta$ becoming of order 1. Finally we evaluate the quantities

$$
r \equiv P_{T} / P_{S}=16 \epsilon_{\bar{N}} \quad n_{S}=1-6 \epsilon_{\bar{N}}+2 \eta_{\bar{N}}
$$

where $\bar{N}$ is the number of efolds that corresponds to a certain cosmological scale. We take $\bar{N}=60$ although the precise number depends on the history of the evolution subsequent to inflation. The results are shown in Fig. 4. ${ }^{2}$

The above scenario assumes that a mechanism for the field $\Phi$ to relax to its minimum at late time can be found. Such minimum has to be located at scales $\Phi \ll M$, otherwise the value of the Planck mass during inflation and the present values would differ and we would lose the connection between the scale of inflation and the value of $V\left(\chi_{0}\right) / M_{P l}^{4}$, obtained through the standard model effective potential with the parameters measured today. This would make the problem of overproduction of gravity waves even more severe, since the relevant quantity during inflation in this case would be even bigger than the value $V\left(\chi_{0}\right) / M_{P l}^{4}$ which we can compute today from the effective potential.

\footnotetext{
${ }^{2}$ Note that in Ref. [1] the calculations were obtained in an expansion for $\Phi \ll M$ for the slow-roll parameters, which does not hold for very small $\gamma_{4}$, therefore leading to incorrect results for $\gamma_{4} \ll 10^{-4}$, which corresponds to a sizable $r$ in Fig. 4 .
}

Generically moreover the condition that at late times $\Phi \ll M$ would also guarantee that we recover Einstein gravity (i.e., that we can satisfy fifth force constraints and also that the Planck mass is not varying rapidly with time [19]). Actually the condition on the final value for $\Phi$ is that, before nucleosynthesis, we reach $f \approx 1$, which in the case of having only the coupling $\gamma_{4} \neq 0$ reduces to the condition $\phi / M \ll \gamma_{4}^{-1 / 4}$. In order to achieve such relaxation to small values we may introduce a potential and a convenient choice seems to introduce it directly in the Einstein frame as a potential $U(\Phi)$. At the same time we have to be careful because we do not want such potential to become dominant during inflation and we do not want such potential to induce extra stages of inflation after the tunneling transition, otherwise our conclusions would be changed. Such an extra inflationary stage can actually arise if we introduce a polynomial potential $U(\Phi) \propto \Phi^{n}$, since after tunneling we are working at values $\Phi \gg M$ and so the slow-roll conditions would be satisfied. In order to avoid such complications we introduce here as an example an exponential potential which does not lead to additional inflation but to a rapid evolution for $\Phi$ to small values. The potential that we consider is of the form $U(\Phi)=\mu^{4}\left(e^{s \Phi}-1\right)$, where $\mu$ is a mass scale and $s$ is a parameter, which we will choose as an explicit example equal to 2 . Interestingly we observe that such potential also arises from a simple power law 
potential $U_{J}(\phi) \propto \phi^{n}$ in the Jordan frame, where $n$ is a large exponent: in fact after a frame transformation $U=$ $U_{J} /(1+f)^{2}$ we have checked numerically that for $\Phi \gg 1$ this corresponds to an Einstein frame potential of the form $U(\Phi) \propto e^{s \Phi}$, where we find the correspondence $s=(n-8) / 5$. Note that for $n=8$ we would get a flat potential (because the denominator $(1+f)^{2}$ and the numerator would have the same power) and instead we recover the $e^{2 \Phi}$ case for $n=18$. Note also that at small $\phi$ the subtraction of zero point energy is automatically obtained with a power law $U_{J}$. Using now the Einstein frame potential the scale $\mu$ has to be chosen such that at the tunneling epoch, for a field value of $\Phi=\Phi_{T}$, the potential $U\left(\Phi_{T}\right) \ll \bar{V}$. Typical values for $\Phi_{T}$ are at least of order 20-60 (depending on the value of $\gamma_{4}$ and on the precise time at which the tunneling transition happens). We show in Fig. 5 of the Appendix how the field evolves in the presence of a radiation component in an example, starting from the tunneling time until nucleosynthesis, showing that the condition $f \approx 1$ can be reached and fifth force bounds can be satisfied. Note that, in cases in which the field has not completely yet relaxed to zero, there can be a tiny timedependent correction to the Planck mass, which can lead to interesting phenomenology. We leave however more detailed discussions for further analysis.

In conclusion we have shown that a mechanism in which the energy density for inflation is provided by the Higgs field starting in a shallow false minimum can be compatible with observations only if $m_{H} \lesssim 124 \mathrm{GeV}$ (and also $m_{t}$ has to be smaller than about $171 \mathrm{GeV}$ ). This is significantly lower than experimental observations unless the theoretical error on the RGE, of about $0.3 \mathrm{GeV}$ on $m_{H}$ according to $[6,7]$, is largely underestimated. We have also shown that introducing a large nonminimal coupling of the Higgs of order $10^{2}-10^{3}$ can push this upper bound on larger values, closer to present observations. Such bounds could be also be evaded by modifying the shape of the potential in a model-dependent way. For instance a possibility would be to introduce direct couplings of the Higgs with the extra scalar as in [30], which would change the potential either at tree level or in the RGE, or perhaps to allow a faster change of the barrier in hybrid models during inflation (see [15]).

\section{Acknowledgments}

We thank A. Strumia and F. Bezrukov for useful discussions.

\section{APPENDIX}

We numerically solve in this appendix the evolution equations after tunneling which describe: (i) the Higgs field which starts from a value $\chi_{T}<\chi_{0}$, then quickly evolves toward its minimum and decays with a rate $\Gamma_{\chi}$ into radiation and finally relaxes at the usual electroweak vacuum $v$, (ii) a radiation component with energy density $\rho_{R}$ sourced by the
Higgs field and simply diluted as $a(t)^{-4}$, (iii) the field $\Phi$ in the Einstein frame which evolves under the potential $V_{T} \equiv \frac{V(\chi)}{f(\Phi)^{2}}+U(\Phi)$ which contains both the Einstein frame Higgs potential and a potential $U(\Phi)$ assumed to be subdominant before tunneling (iiii) the metric with expansion rate $H$ which is sourced by the sum of the energy densities of such components:

$$
\begin{array}{r}
\ddot{\chi}+3 H \dot{\chi}+\frac{\partial V_{T}}{\partial \chi}+\Gamma_{\chi} \dot{\chi}=0 \\
\dot{\rho}_{R}+4 H \rho_{R}-\Gamma_{\chi} \dot{\chi}^{2}=0 \\
\ddot{\Phi}+3 H \dot{\Phi}+\frac{\partial V_{T}}{\partial \Phi}=0 \\
H^{2}=\frac{V_{T}+\frac{\dot{\chi}^{2}}{2}+\frac{\dot{\Phi}^{2}}{2}+\rho_{R}}{3 M^{2}}
\end{array}
$$

The decay rate of Higgs into other particles is large (since the couplings in the SM are large at high field values) and so the field should relax very quickly to zero, resulting in overdamped oscillations. Modeling the decay rate exactly is a very tough problem in a field dependent nonequilibrium situation, however our conclusions are not very much dependent on the details of reheating, since we are interested in the evolution of $\Phi$, so we assume simply a large constant decay rate (we choose in the example the value $\left.\Gamma_{\chi}=10^{-2} \chi_{T}\right)$. As we said we also assume $U(\Phi)=\mu^{4}\left(e^{s \Phi}-1\right)$. We analyze first the example $s=2, \mu=10^{8} \mathrm{GeV}$ and we show in Fig. 5, that $\Phi$ rolls to small values, so that $f \approx 1$, when the temperature is still well above nucleosynthesis. A quantity we are interested in is the following

$$
\alpha=-2 \frac{d \log f(\Phi)}{d \Phi}
$$

which is linked to the PPN parameters [19]: the experimental bounds are satisfied if $\alpha^{2} \ll 10^{-3}$, and this is shown also in Fig. 5. We also define a temperature given approximately as $T \equiv\left(\rho_{R} / g_{*}\right)^{1 / 4}$, where we use $g_{*}=10^{2}$. The parameter $\mu$ is bounded since we want $\mu^{4} e^{s \Phi_{T}} \ll V_{0}$, which leads to $\mu \ll$ $\left(10^{11}-10^{13}\right) \mathrm{GeV}$ for $s=2$. At the same time $\mu$ has to be also large enough otherwise the field would not roll down toward its minimum before nucleosynthesis. Note that $\Phi$ tracks the radiation component and the expansion goes like $a \propto t^{1 / 2}$. Such an evolution is intriguing because at late time it may show up as a nontrivial extra radiation component in the energy budget of the Universe and it may also lead to small variations of the Planck mass at late time. Moreover, note that adding a small mass term $m_{\Phi} \Phi^{2}$ to $U$ it is also possible to have a late time behavior for $\Phi$ with cold dark matter properties if $m_{\Phi}$ is of the order of $H$ at matter radiation equality, $\mathcal{O}(e \mathrm{~V})$. Note that for larger values of $s$ the behavior is always tracking radiation, with a smaller relative 
energy density for increasing $s$. For smaller values of $\mu$ clearly the evolution is slower and takes place at a later time.

Note that instead if $s<2$ we have a scalar-field dominated evolution in which $a(t)$ grows faster than during radiation. For instance in the case $s=1$ we have $a(t) \propto t^{2}$ and this produces about 20-30 efolds of extra accelerated evolution during which the perturbations are not reentering the horizon, and this should be taken into account in the calculation of cosmological observables, such as $n_{s}$ and $r$. In such cases it is compulsory to have also an additional mass term and a decay rate in order to get rid of such an extra component before nucleosynthesis.

We stress that there is room for wide variations in parameter space and so we leave this for a more detailed future work.
[1] I. Masina and A. Notari, The higgs mass range from standard model false vacuum inflation in scalar-tensor gravity, Phys. Rev. D 85, 123506 (2012).

[2] I. Masina and A. Notari, Standard Model False Vacuum Inflation: Correlating the Tensor-to-Scalar Ratio to the Top Quark and Higgs Boson Masses, Phys. Rev. Lett. 108, 191302 (2012).

[3] I. Masina and A. Notari, Inflation from the Higgs field false vacuum with hybrid potential, J. Cosmol. Astropart. Phys. 11 (2012) 031.

[4] N. Cabibbo, L. Maiani, G. Parisi, and R. Petronzio, Bounds on the fermions and higgs boson masses in grand unified theories, Nucl. Phys. B158, 295 (1979); P. Q. Hung, Vacuum Instability and New Constraints on Fermion Masses, Phys. Rev. Lett. 42, 873 (1979); M. Lindner, Implications of triviality for the standard model, Z. Phys. C 31, 295 (1986); M. Lindner, M. Sher, and H. W. Zaglauer, Probing vacuum stability bounds at the fermilab collider, Phys. Lett. B 228, 139 (1989); M. Sher, Electroweak higgs potentials and vacuum stability, Phys. Rep. 179, 273 (1989); B. Schrempp and M. Wimmer, Top quark and higgs boson masses: Interplay between infrared and ultraviolet physics, Prog. Part. Nucl. Phys. 37, 1 (1996).

[5] J. A. Casas, J. R. Espinosa, and M. Quiros, Standard model stability bounds for new physics within LHC reach, Phys. Lett. B 382, 374 (1996); G. Isidori, G. Ridolfi, and A. Strumia, On the metastability of the standard model vacuum, Nucl. Phys. B609, 387 (2001); N. Arkani-Hamed, S. Dubovsky, L. Senatore, and G. Villadoro, (No) eternal inflation and precision Higgs physics, J. High Energy Phys. 03 (2008) 075.

[6] G. Degrassi, S. Di Vita, J. Elias-Miro, J. R. Espinosa, G. F. Giudice, G. Isidori, and A. Strumia, Higgs mass and vacuum stability in the Standard model at NNLO, J. High Energy Phys. 08 (2012) 098.

[7] D. Buttazzo, G. Degrassi, P. P. Giardino, G. F. Giudice, F. Sala, A. Salvio, and A. Strumia, Investigating the nearcriticality of the higgs boson, J. High Energy Phys. 12 (2013) 089.

[8] F. Bezrukov, M. Y. Kalmykov, B. A. Kniehl, and M. Shaposhnikov, Higgs boson mass and new physics, J. High Energy Phys. 10 (2012) 140.

[9] T. Biswas and A. Notari, Can inflation solve the hierarchy problem?, Phys. Rev. D 74 (2006) 043508.
[10] F. Di Marco and A. Notari, Graceful old inflation, Phys. Rev. D 73 (2006) 063514.

[11] (ATLAS Collaboration), Combined search for the standard model higgs boson using up to $4.9 \mathrm{fb}^{-1}$ of pp collision data at $\sqrt{s}=7 \mathrm{TeV}$ with the ATLAS detector at the LHC, Phys. Lett. B 710, 49 (2012); Combined Search for the Standard Model Higgs Boson Using up to $4.9 \mathbf{f b}^{-1}$ of pp Collision Data at $\sqrt{s}=7 \mathbf{T e V}$ with the ATLAS Detector at the LHCPhys. Rev. Lett. 108, 111803 (2012); (CMS Collaboration), Search for the Standard Model Higgs boson in the decay channel $\boldsymbol{H} \rightarrow \boldsymbol{Z} \boldsymbol{Z}^{(*)} \rightarrow 4 \boldsymbol{l}$ with $4.8 \mathbf{f b}^{-1}$ of $\boldsymbol{p p}$ collision data at $\sqrt{s}=7 \mathbf{T e V}$ with ATLAS, Phys. Lett. B 710, 383 (2012).

[12] (TEVNPH Working Group and CDF for the and D0 Collaborations), Combined CDF and D0 search for standard model higgs boson production with up to $10.0 \mathrm{fb}^{-1}$ of data, arXiv:1203.3774.

[13] P. A. R. Ade et al. (BICEP2 Collaboration), Detection of BMode Polarization at Degree Angular Scales by BICEP2, Phys. Rev. Lett. 112, 241101 (2014).

[14] P. A. R. Ade et al. (BICEP2 and Planck Collaborations), A joint analysis of BICEP2/Keck array and planck data, arXiv:1502.00612.

[15] M. Fairbairn, P. Grothaus, and R. Hogan, The problem with false vacuum Higgs inflation, arXiv:1403.7483.

[16] J. Beringer et al. (Particle Data Group), Review of particle physics, Phys. Rev. D 86, 010001 (2012) and 2013 partial update for the 2014 edition.

[17] T. ATLAS et al. (D0 Collaboration), First combination of tevatron and LHC measurements of the top-quark mass, arXiv:1403.4427.

[18] S. R. Coleman, The fate of the false vacuum. 1. semiclassical theory, Phys. Rev. D 15, 2929 (1977); 16, 1248(E) (1977).

[19] C. M. Will, The confrontation between general relativity and experiment, Living Rev. Relativity 9, 3 (2005).

[20] I. Masina, Gravitational wave background and higgs false vacuum inflation, Phys. Rev. D 89, 123505 (2014).

[21] M. Fairbairn, P. Grothaus, and R. Hogan, The problem with false vacuum higgs inflation, J. Cosmol. Astropart. Phys. 06 (2014) 039.

[22] K. A. Olive et al. (Particle Data Group), Review of particle physics, Chin. Phys. C, 38, 090001 (2014)

[23] F. Bezrukov and M. Shaposhnikov, Higgs inflation at the critical point, Phys. Lett. B 734, 249 (2014). 
[24] Y. Hamada, H. Kawai, K. y. Oda, and S. C. Park, Higgs Inflation is Still Alive after the Results from BICEP2, Phys. Rev. Lett. 112, 241301 (2014).

[25] F. L. Bezrukov and M. Shaposhnikov, The standard model higgs boson as the inflaton, Phys. Lett. B 659 , 703 (2008).

[26] J. L. F. Barbon and J. R. Espinosa, On the naturalness of higgs inflation, Phys. Rev. D 79, 081302 (2009).

[27] C. P. Burgess, H. M. Lee, and M. Trott, On Higgs inflation and naturalness, J. High Energy Phys. 07 (2010) 007.
[28] F. Bezrukov, A. Magnin, M. Shaposhnikov, and S. Sibiryakov, Higgs inflation: consistency and generalisations, J. High Energy Phys. 01 (2011) 016.

[29] A. Kehagias and A. Riotto, Remarks about the tensor mode detection by the BICEP2 collaboration and the super-planckian excursions of the inflaton Field, Phys. Rev. D 89, 101301 (2014).

[30] J. Elias-Miro, J. R. Espinosa, G. F. Giudice, H. M. Lee, and A. Strumia, Stabilization of the electroweak vacuum by a scalar threshold effect, J. High Energy Phys. 06 (2012) 031. 